\title{
THE STABLE SHAPE OF COMPACT SPACES WITH COUNTABLE COHOMOLOGY GROUPS
}

\author{
SŁAWOMIR NOWAK \\ University of Warsaw, Poland \\ Dedicated to Professor Sibe Mardešić on the occasion of his 80th birthday \\ Abstract. Let $X$ be a compact Hausdorff space and $q \in \mathbb{Z}$ be an \\ integer such that the integral cohomology groups $H^{n}(X ; \mathbb{Z})$ are countable \\ for $n<q$ and the stable cohomotopy groups $\pi_{s}^{n}(X)$ of $X$ are countable for \\ $n \geq q$. Then there exists a compact metrizable compact space $Y$ with the \\ same stable shape as $X$.
}

The stable shape category ShStab ([Mi, Mi-Se $\left.1, \mathrm{Mi}_{-} \mathrm{Se}_{2}, \mathrm{~N}_{1}, \mathrm{~N}_{2}\right]$ ) can be obtained as a modification of the classical shape category ([Bo, Ma-Se]) when we replace the homotopy classes of maps by the stable homotopy classes of maps. Since the Čech cohomology functors (defined on the homotopy category of compact Hausdorff spaces) factorize via ShStab, it is especially useful when studying cohomological properties of a space. From this point of view it is important to know when a given compact space $X$ can be replaced (up to stable shape) by a simpler one. In particular in $\left[\mathrm{N}_{2}\right]$ it has been shown that $X$ has the stable shape of a compact metrizable space $Y$ if and only if the stable cohomotopy groups $\pi_{s}^{n}(X)$ are countable for $n \in \mathbb{Z}$.

Here we prove that such a compact metric space $Y$ exists iff there is an index $p$ such that $H^{n}(X ; \mathbb{Z})$ are countable for $n \leq p$ and $\pi_{s}^{n}(X)$ are countable for $k>p$.

The Cartesian product of uncountably many copies of the Adams-KahnTaylor continuum [Ma-Se, p. 284] is an acyclic and non-movable compact space which does not have the stable shape of any metric space.

In the movable case $\left[\mathrm{N}_{2}\right.$, Theorem $\left.\mathrm{B}\right]$ vanishing the groups $H^{n}(X ; \mathbb{Z})$ for $n>0$ (or respectively for almost all $n$ ) implies that $X$ has the stable shape

2000 Mathematics Subject Classification. 55P55, 55P40, 55N05, 55Q07.

Key words and phrases. Stable cohomotopy groups, stable shape. 
of a 0-dimensional space (or that the $k$-fold suspension of $X$ has the stable shape of a finite dimensional space).

Hence it seems natural to presume that a compact movable space $X$ has the stable shape of a metric space iff the groups $H^{n}(X ; \mathbb{Z})$ are countable for every $n$, but the question whether it is true or not is still open.

\section{Preliminaries}

A spectrum $\mathbb{E}=\left\{E_{n}, \varepsilon_{n}\right\}$ is a sequence of spaces and maps $\varepsilon_{n}: \mathbf{S}\left(E_{n}\right) \rightarrow$ $E_{n+1}$. It is a $\mathrm{CW}$ spectrum if $E_{n}$ are $\mathrm{CW}$ complexes and $\varepsilon_{n}$ is a cellular inclusion.

If $\mathbb{E}=\left\{E_{n}, \varepsilon_{n}\right\}$ and $\mathbb{F}=\left\{F_{n}, \varepsilon_{n}^{\prime}\right\}$ are arbitrary spectra, then a Whitehead map ( $W$-map) f $: \mathbb{E} \rightarrow \mathbb{F}$ is a sequence of maps $f_{n}: E_{n} \rightarrow F_{n}$ such that the diagram

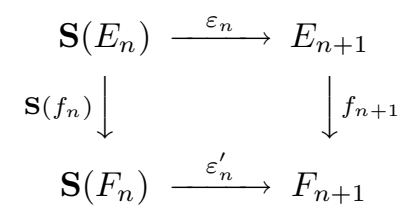

commutes up to homotopy (compare [K-K-S, p. 213]).

We say that $\mathbf{f}$ is a function of spectra if the diagram (1.1) commutes strictly.

As for prerequisites, the reader is expected to be familiar with the basic concepts of the stable homotopy theory ([A, Ma, Sw]). In particular, a knowledge of the notions of maps, morphisms, cells and $r$-dimensional skeletons of CW spectra is assumed and we use also the homology and homotopy groups of CW spectra (see [Ma] or [Sw, Chapters 8-14]).

For arbitrary spectrum $\mathbb{E}=\left\{E_{n}, \varepsilon_{n}\right\}$ we may define the $n$-dimensional homotopy group $\pi_{n}(\mathbb{E})$ as the direct limit of the direct sequence $\{\ldots \rightarrow$ $\left.\pi_{n+k}\left(E_{k}\right) \rightarrow \pi_{n+k+1}\left(E_{k+1}\right) \rightarrow \ldots\right\}$, where the middle arrow is the composition of the homorphism $\pi_{n+k}\left(E_{n+k}\right) \rightarrow \pi_{n+k+1}\left(\mathbf{S}\left(E_{n+k}\right)\right)$ induced by the suspension and the homorphism $\pi_{n+k+1}\left(\varepsilon_{n+k}\right): \pi_{n+k+1}\left(\mathbf{S}\left(E_{n+k}\right)\right) \rightarrow$ $\pi_{n+k+1}\left(E_{n+k+1}\right)$.

REMARK 1.1. If $\mathbb{E}$ is a $\mathrm{CW}$ spectrum and $\mathbf{f}: \mathbb{E} \rightarrow \mathbb{F}$ is a $\mathrm{W}$-map, then there exists ([Sw, Lemma 10.4] and [K-K-S, p. 213]) a function of spectra $\mathbf{g}: \mathbb{E} \rightarrow \mathbb{F}$ such that $\mathbf{f}$ and $\mathbf{g}$ are homotopic as W-maps.

Each W-map f : $\mathbb{E} \rightarrow \mathbb{F}$ induces homorphism $\pi_{n}(\mathbf{f}): \pi_{n}(\mathbb{E}) \rightarrow \pi_{n}(\mathbb{F})$ of the homotopy groups ([Sw, Ma $]$ ) of spectra. If $\mathbf{f}$ and $\mathbf{g}$ are homotopic, then $\pi_{n}(\mathbf{f})=\pi_{n}(\mathbf{g})$.

A pair $(\mathbb{E}, \boldsymbol{\alpha})$, where $\boldsymbol{\alpha}: \mathbb{E}_{0} \rightarrow \mathbb{F}$ is a function defined on a cofinal subspectrum $\mathbb{E}_{0}$ of a $C W$ spectrum $\mathbb{E}$, is called a CW substitute of a spectrum $\mathbb{F}$ iff $\pi_{n}(\boldsymbol{\alpha})$ is an isomorphism for every index $n$. When no confusion can arise 
we ignore the second element of the pair $(\mathbb{E}, \boldsymbol{\alpha})$ and we say that $\mathbb{E}$ is a $\mathrm{CW}$ substitute of $\mathbb{F}$.

Remark 1.2. For every spectrum there exists a CW substitute [K-K-S, Proposition 2.3].

Suppose that $X$ is a compact Hausdorff space. By $\mathbb{F}(X)=\left\{\left(\operatorname{Map}\left(X, S^{n}\right)\right.\right.$, $\left.\left.\lambda_{n}\right)\right\}$ we denote the function spectrum of $X$, where $\lambda_{n}: \mathbf{S}\left(\operatorname{Map}\left(X, S^{n}\right)\right) \rightarrow$ $\operatorname{Map}\left(X, S^{n+1}\right)$ is defined by the formula $\lambda_{n}(f \wedge t)(x)=f(x) \wedge t$ for every $f \wedge t \in \operatorname{Map}\left(X, S^{n}\right) \wedge S^{1}=\mathbf{S}\left(\operatorname{Map}\left(X, S^{n}\right)\right)$.

Proposition 1.3 ([ $\mathrm{N}_{2}$, Corollary 6.1.2]). For every compact Hausdorff space $X$ and every $C W$ substitute $\mathbb{W}_{X}$ of $\mathbb{F}(X)$ we have $\pi_{s}^{-n}(X) \cong \pi_{n}\left(\mathbb{W}_{X}\right)$ and $H^{-n}(X ; \mathbb{Z}) \cong H_{n}\left(\mathbb{W}_{X} ; \mathbb{Z}\right)$, where $n \in \mathbb{Z}$.

\section{Spectra with countable Skeletons}

We adhere to the convention that the $r$-dimensional skeleton ( $r$-skeleton) $E^{(r)}$ of a $\mathrm{CW}$ complex $E$ (or $r$-skeleton $\mathbb{E}^{(r)}$ of a $\mathrm{CW}$ spectrum $\mathbb{E}$ ) is also defined for the case when $r=\infty$ and $E^{(\infty)}=E\left(\right.$ or $\left.\mathbb{E}^{(\infty)}=\mathbb{E}\right)$.

Lemma 2.1. Let $q \in \mathbb{Z}$ or $q=\infty$ and $\mathbf{f}=\left\{f_{n}\right\}: \mathbb{E}=\left\{E_{n}, \varepsilon_{n}\right\} \rightarrow \mathbb{F}$ be a $W$-map such that the following conditions are satisfied:

(1) $\mathbb{F}$ is a $C W$ spectrum,

(2) $f_{n}: E_{n} \rightarrow F_{n}$ is a homotopy equivalence,

(3) $E_{n}$ is a $C W$ complex such that the $r$-skeleton $E_{n}^{(r)}$ of $E_{n}$ is a countable $C W$ complex, where $r=\max (n+q, 0)$,

(4) $\varepsilon_{n}: \mathbf{S}\left(E_{n}\right) \rightarrow E_{n+1}$ is a cellular map.

Then there exists a $C W$ spectrum $\mathbb{G}=\left\{G_{n}\right\}$ and a function of spectra $\mathbf{h}=$ $\left\{h_{n}\right\}: \mathbb{G} \rightarrow \mathbb{F}$, which induces an isomorphism of spectra. The q-skeleton of $\mathbb{G}$ is a countable spectrum.

Proof. It is known [Sw, Proposition 8.3] that for every spectrum $\mathbb{E}$ satisfying the condition (4) there exists a function of spectra $\mathbf{g}=\left\{g_{n}\right\}: \mathbb{G} \rightarrow \mathbb{F}$, where $g_{n}: G_{n} \rightarrow E_{n}$ is a homotopy equivalence. The $n$th term $G_{n}$ of $G$ is obtained as the telescope of the sequence $\mathbf{S}^{n}\left(E_{0}\right) \stackrel{\mathbf{S}^{n}\left(\varepsilon_{0}\right)}{\longrightarrow} \mathbf{S}^{n-1}\left(E_{1}\right) \stackrel{\mathbf{S}^{n-1}\left(\varepsilon_{0}\right)}{\longrightarrow}$ $\mathbf{S}^{n-2}\left(E_{2}\right) \rightarrow \ldots \mathbf{S}\left(E_{n-1}\right) \stackrel{\varepsilon_{n}}{\longrightarrow} E_{n}$, i.e., the union of the reduced mapping cylinders $M_{i}$ of $\mathbf{S}^{n-i}\left(\varepsilon_{i}\right)$, where the top of $M_{i}$ is identified with the bottom of $M_{i+1}$ and $i \geq 1$. The construction guarantees that the $(q+n)$-skeleton of $G_{n}$ and the $q$-skeleton of $\mathbb{G}$ are countable.

Lemma 2.2. Let $q \in \mathbb{Z}$ or $q=\infty$ and suppose that $\mathbb{E}$ is a $C W$ spectrum such that $\pi_{k}(\mathbb{E})$ is a countable group for $k<q+1$. Then there exist a $C W$ spectrum $\mathbb{G}$ and an isomorphism of spectra $\mathbf{f}: \mathbb{E} \rightarrow \mathbb{G}$ such that the q-skeleton $\mathbb{G}^{(q)}$ of $\mathbb{G}$ is a countable spectrum. 
Proof. We may assume $[\mathrm{A}$, p. 150$]$ that $\mathbb{E}$ is an $\Omega_{0}$ spectrum. Hence $\pi_{n}\left(E_{r}\right)=\pi_{n-r}(\mathbb{E})$ for $n \geq 1$. By [W, Theorem $\mathrm{C}$ ] there exists a CW complex $F_{n}$ and a cellular homotopy equivalence $f_{n}: F_{n} \rightarrow E_{n}$ such that $F_{n}$ has a countable $(n+q)$-skeleton.

Let $\varepsilon_{n}=g_{n+1}^{-1} \mathbf{S}\left(f_{n}\right): \mathbf{S}\left(F_{n}\right) \rightarrow F_{n+1}$, where $g_{n+1}^{-1}: E_{n+1} \rightarrow F_{n+1}$ is a homotopy inverse of $f_{n+1}$. Then $\left(F_{n}, \varepsilon_{n}\right)$ is a spectrum and $\left\{f_{n}\right\}: \mathbb{E} \rightarrow$ $\left(F_{n}, \varepsilon_{n}\right)$ is a W-map. Lemma 2.2 is a consequence of Lemma 2.1.

LEMMA 2.3. For every $C W$ spectrum $\mathbb{W}$ with countable q-skeleton there exists a $C W$ spectrum $\mathbb{V}$ and a morphism of spectra $\mathbf{f}: \mathbb{W} \rightarrow \mathbb{V}$ such that

(1) $\pi_{n}(\mathbf{f}): \pi_{n}(\mathbb{W}) \rightarrow \pi_{n}(\mathbb{V})$ is an isomorphism for $n \leq q$,

(2) $\pi_{n}(\mathbb{V})=0$ for $n>q$,

(3) $\mathbb{V}$ is countable.

Proof. For every CW spectrum $\mathbb{W}$ one can find ([Ma, p. 42, the proof of Proposition 8]) a CW spectrum $\mathbb{W}[-\infty, q]$ and a morphism of spectra $\mathbf{g}$ : $\mathbb{W} \rightarrow \mathbb{W}[-\infty, q]$ such that $\pi_{n}(\mathbf{g}): \pi_{n}(\mathbb{W}) \rightarrow \pi_{n}(\mathbb{W}[-\infty, q])$ is an isomorphism for $n \leq q$ and $\pi_{n}(\mathbb{W}[-\infty, q])=0$ for $n>q$.

This means that the homotopy group $\pi_{n}(\mathbb{W}[-\infty, q])$ of $\mathbb{W}[-\infty, q]$ is countable for every $n$ and by Lemma 2.2 there is an isomorphism $\mathbf{h}: \mathbb{W}[-\infty, q] \rightarrow \mathbb{V}$, where $\mathbb{V}$ is a countable $\mathrm{CW}$ spectrum. The morphism $\mathbf{f}=\mathbf{h g}: \mathbb{W} \rightarrow \mathbb{V}$ satisfies the required conditions.

\section{The MAIN THEOREM}

THEOREM 3.1. Suppose that $X$ is a compact Hausdorff space and $p$ is an integer such that the following conditions are satisfied:

(1) $\pi_{s}^{n}(X)$ is countable for every $n \geq p$,

(2) $H^{n}(X ; \mathbb{Z})$ is countable for every $n<p$.

Then there exists a compactum $Y$ with the same stable shape as $X$.

Proof. We know that

$$
\pi_{s}^{n}(X) \text { is a countable Abelian group }
$$

for $n \geq p=-q$ and $H^{n}(X ; \mathbb{Z})$ is a countable Abelian group for $n<p=-q$.

It is known (see $\left[\mathrm{N}_{2}\right]$ ) that if the condition (3.1) is satisfied for every $n$ then there is a metric compact space $Y$ with $\operatorname{ShStab}(X)=\operatorname{ShStab}(Y)$.

In order to prove theorem it suffices to show that the condition (3.1) is satisfied for $n \geq p-1$. Indeed, for each $n<p$ we can repeat the proceeding argument how many times as it is necessary and we can prove that $\pi_{s}^{n}(X)$ is countable, which gives that (3.1) is satisfied for every $n$.

Let $\boldsymbol{\alpha}: \mathbb{W} \rightarrow \mathbb{F}(X)$ be a CW substitute of $\mathbb{F}(X)$.

Then

$$
H_{n}(\mathbb{W} ; \mathbb{Z}) \text { is countable for } n>q \text { and } \pi_{n}(\mathbb{W}) \text { is countable for } n \leq q .
$$


By Lemma 2.1 we may assume that the $q$-skeleton of $\mathbb{W}$ is countable and that $H_{n}(\mathbb{W} ; \mathbb{Z})$ is a countable Abelian group for $n \leq q$. From Lemma 2.3 we conclude that there is a countable $\mathrm{CW}$ spectrum $\mathbb{V}$ and a morphism of spectra $\mathbf{f}: \mathbb{W} \rightarrow \mathbb{V}$ such that $\pi_{n}(\mathbf{f}): \pi_{n}(\mathbb{W}) \rightarrow \pi_{n}(\mathbb{V})$ is an isomorphism for $n \leq q$ and $\pi_{n}(\mathbb{V})=0$ for $n>q$. Hence

$$
\pi_{n}(\mathbb{V}) \text { and } H_{n}(\mathbb{V} ; \mathbb{Z}) \text { are countable for every } n .
$$

We have the following long exact sequences

$$
\ldots \pi_{n+1}(\mathbb{V}) \rightarrow \pi_{n+1}(\mathbb{E}) \rightarrow \pi_{n}(\mathbb{W}) \rightarrow \pi_{n}(\mathbb{V}) \rightarrow \pi_{n}(\mathbb{E}) \rightarrow \pi_{n-1}(\mathbb{W}) \rightarrow \ldots
$$

and

$$
\begin{aligned}
\ldots H_{n+1}(\mathbb{V} ; \mathbb{Z}) \rightarrow H_{n+1}(\mathbb{E} ; \mathbb{Z}) \rightarrow H_{n}(\mathbb{W} ; \mathbb{Z}) \\
\quad \rightarrow H_{n}(\mathbb{V} ; \mathbb{Z}) \rightarrow H_{n}(\mathbb{E} ; \mathbb{Z}) \rightarrow H_{n-1}(\mathbb{W} ; \mathbb{Z}) \rightarrow \ldots,
\end{aligned}
$$

where $\mathbb{E}$ denotes the mapping cone of $\mathbf{f}: \mathbb{W} \rightarrow \mathbb{V}$.

The exactness of (3.4) implies that $\pi_{n+1}(\mathbb{E})$ and $\pi_{n}(\mathbb{W})$ are isomorphic for $n>q$ and $\pi_{n}(\mathbb{E})=0$ for $n \leq q+1$. Therefore the spectrum $\mathbb{E}$ is bounded below. By the Hurewicz Theorem for spectra we conclude that $\pi_{q+2}(\mathbb{E})$ and $H_{q+2}(\mathbb{E} ; \mathbb{Z})$ are isomorphic. From (3.2) and (3.3) it follows that the groups $H_{q+1}(\mathbb{W} ; \mathbb{Z})$ and $H_{q+2}(\mathbb{V} ; \mathbb{Z})$ are countable.

If we have an exact sequence $G_{1} \rightarrow G \rightarrow G_{2}$ of Abelian groups, then the countability of $G_{1}$ and $G_{2}$ implies the countability of $G$. Therefore the exactness $(3.5)$ implies that $H_{q+2}(\mathbb{E} ; \mathbb{Z}) \cong \pi_{q+2}(\mathbb{E}) \cong \pi_{q+1}(\mathbb{W}) \cong \pi_{s}^{-q-1}(X)=$ $\pi_{s}^{p-1}(X)$ are countable Abelian groups and the condition (3.1) is satisfied for $n \geq p-1$.

CONJecture 3.2. A movable compact Hausdorff space $X$ has the stable shape of a compact metric space iff the cohomology groups $H^{n}(X ; \mathbb{Z})$ are countable for $n \geq 0$.

\section{REFERENCES}

[A] J. F. Adams, Stable Homotopy and Generalized Homology, University of Chicago Press, Chicago, 1974.

[Bo] K. Borsuk, Theory of Shape, PWN-Polish Scientific Publishers, Warszawa, 1975.

[K-K-S] D. S. Kahn, J. Kaminker and C. Schochet, Generalized homology theories on compact metric spaces, Michigan Math. J. 24 (1977), 203-224.

[Ma-Se] S. Mardešić and J. Segal, Shape Theory, North-Holland Publishing Co., Amsterdam-New York-Oxford 1982.

[Ma] H. R. Margolis, Spectra and the Steenrod Algebra. Modules over the Steenrod algebra and the stable homotopy category, North-Holland Publishing Co., Amsterdam, 1983.

[Mi] T. Miyata, Generalized stable shape and duality, Topology Appl. 109 (2001), 7588.

[Mi-Se 1$]$ T. Miyata and J. Segal, Generalized stable shape and the Whitehead theorem, Topology Appl. 63 (1995), 139-164. 
[Mi-Se 2$]$ T. Miyata and J. Segal, Generalized stable shape and Brown's representation theorem, Topology Appl. 94 (1999), 275-305.

$\left[\mathrm{N}_{1}\right] \quad$ S. Nowak, On the relationships between shape properties of subcompacta of $S^{n}$ and homotopy properties of their complements, Fund. Math. 128 (1987), 47-60.

$\left[\mathrm{N}_{2}\right] \quad$ S. Nowak, Stable cohomotopy groups of compact spaces, Fund. Math. 180 (2003), 99-137.

[Sw] R. M. Switzer, Algebraic Topology - Homotopy and Homology, Springer-Verlag, New York-Heidelberg, 1975.

[W] C. T. C. Wall, Finiteness conditions for CW-complexes, Ann. of Math. (2) 81 (1965), 59-69.

S. Nowak

Institute of Mathematics

University of Warsaw

ul. Banacha 2, 02-097 Warszawa

Poland

E-mail: snowak@mimuw.edu.pl

Received: 11.9 .2006$.

Revised: 26.2.2007. 